Article

\title{
Unsupervised Change Detection Using Fast Fuzzy Clustering for Landslide Mapping from Very High-Resolution Images
}

\author{
Tao Lei ${ }^{1}{ }^{(1)}$, Dinghua Xue ${ }^{1}$, Zhiyong $\mathrm{Lv}^{2}$, Shuying $\mathrm{Li}^{3, *}$, Yanning Zhang ${ }^{4}$ and Asoke K. Nandi ${ }^{5}$ \\ 1 School of Electronical and Information Engineering, Shaanxi University of Science and Technology, \\ Xi'an 710021, China; leitao@sust.edu.cn (T.L.); xdinghua@163.com (D.X.) \\ 2 School of Computer Science and Engineering, Xi'an University of Technology, Xi'an 710048, China; \\ Lvzhiyong_fly@hotmail.com \\ 3 School of Automation, Xi'an University of Posts and Telecommunications, Xi'an 710121, China \\ 4 School of Computer Science, Northwestern Polytechnical University, Xi'an 710072, China; \\ ynzhang@nwpu.edu.cn \\ 5 Department of Electronic and Computer Engineering, Brunel University London, Middlesex UB8 3PH, UK; \\ asoke.nandi@brunel.ac.uk \\ * Correspondence: angle_lisy@163.com; Tel.: +86-029-88166341
}

Received: 9 July 2018; Accepted: 27 August 2018; Published: 30 August 2018

\begin{abstract}
Change detection approaches based on image segmentation are often used for landslide mapping (LM) from very high-resolution (VHR) remote sensing images. However, these approaches usually have two limitations. One is that they are sensitive to thresholds used for image segmentation and require too many parameters. The other one is that the computational complexity of these approaches depends on the image size, and thus they require a long execution time for very high-resolution (VHR) remote sensing images. In this paper, an unsupervised change detection using fast fuzzy c-means clustering (CDFFCM) for LM is proposed. The proposed CDFFCM has two contributions. The first is that we employ a Gaussian pyramid-based fast fuzzy c-means (FCM) clustering algorithm to obtain candidate landslide regions that have a better visual effect due to the utilization of image spatial information. The second is that we use the difference of image structure information instead of grayscale difference to obtain more accurate landslide regions. Three comparative approaches, edge-based level-set (ELSE), region-based level-set (RLSE), and change detection-based Markov random field (CDMRF), and the proposed CDFFCM are evaluated in three true landslide cases in the Lantau area of Hong Kong. The experiments show that the proposed CDFFCM is superior to three comparative approaches in terms of higher accuracy, fewer parameters, and shorter execution time.
\end{abstract}

Keywords: landslide mapping (LM); change detection; image segmentation; fuzzy c-means (FCM) clustering

\section{Introduction}

Change detection is used to detect the changed information of the target area by analyzing the multi-temporal images acquired in different periods of the same geographical area. With the rapid development of remote sensing technology, change detection has become an important research topic in the field of remote sensing, and has also been widely used in resource and environmental surveys [1], environmental monitoring [2], urban expansion and change information acquisition [3], land use [4], fire area survey and disaster detection and assessment [5], etc. For a geological disaster, researchers can obtain disaster information by analyzing the changed information of multi-temporal 
remote sensing images without entering the disaster scene. Because landslides seriously endanger people's property and safety, it is important to study automatic approaches of landslide identification and apply these approaches to mark the location and range of the landslide for mitigating potential harm, assisting post-disaster rescue, and rebuilding. Automatic approaches to landslide identification can quickly provide detailed landslide information including the landslide site, the range, and the degree of disaster without entering the disaster scene. According to the obtained landslide information, rescue and reconstruction work can be effectively carried out to reduce the degree of disaster and personal safety threats and improve work efficiency.

As early techniques used for landslide identification rely on visual interpretation, they are highly labor-intensive and time-consuming. Existing techniques always employ different change detection approaches that compute the difference between pre- and post-event images to achieve automatic landslide identification. The popular change detection approaches can be categorized into three groups: threshold segmentation-based approaches, approaches based on the combination of feature extraction and clustering, and region segmentation-based approaches.

The first group of approaches, i.e., threshold segmentation-based change detection, first computes the difference image of bitemporal images, and then obtains changed regions using a threshold segmentation technique. Nelson [6] proposed a single-threshold approach used for change detection. Although the approach has a low computational complexity, it is sensitive to noise, and it provides a low accuracy for LM. To improve the single-threshold approach, Jin [7] proposed a double-threshold segmentation approach and applied it to change detection. Although the double-threshold approach is superior to the single-threshold method for improving the accuracy of change detection, the choice of thresholds is still quite difficult. Moreover, the method has a low robustness for change detection. Based on the previous research, Lv et al. [8] first computes the difference image by employing multi-threshold segmentation and voting strategy. After that, a region-based image segmentation method is used for obtaining a superpixel result of the post-event image. Finally, the changed regions are obtained by computing the ratio of the number of pixels belonging to the foreground or background. The multi-threshold segmentation is able to provide better results for change detection, but it has a high computational complexity and requires more parameters. Clearly, change detection approaches based on threshold segmentation $[9,10]$ are simple and fast, but they generate changed regions only depending on the grayscale value of images. They cannot obtain accurate detection results because the statistical features of image and spatial information are missing. Therefore, it is difficult to obtain accurately changed regions using threshold approaches.

Compared to the first group of approaches used for change detection, the second utilizes the combination of feature transformation and clustering to achieve change detection. Dong et al. [11] proposed a change detection approach based on the combination of discrete wave transform (DWT) and fuzzy c-means (FCM). First, DWT is used to obtain the frequency feature of bitemporal images. The changed regions are generated by computing the minus value and ratio value of images transformed by DWT. After that, the lower-half frequency components of the minus image and the higher-half frequency components of the ratio image are fused to enhance the contrast of the changed regions and the unchanged regions. The inverse discrete wave transform (IDWT) is then used to generate the final difference image. Finally, FCM is used to obtain changed regions. However, DWT only extracts image features with a single-scale and a single-phase. Because Gabor is able to extract image features of multi-scale and multi-phases, it is more popular than DWT for image transformation. Li et al. [12] employ Gabor to extract the texture features of an image instead of DWT. Then, the difference image is obtained by computing the difference of bitemporal images processed by Gabor. Finally, a clustering method is used to extract the changed regions. Because the local spatial feature of images is exploited, the approach is superior to DWT-based approaches for change detection. To obtain a better difference image, Zheng et al. [13] applied a logarithm operation to the ratio of bitemporal images to improve the difference image. Moreover, the saliency map of a post-event image is used for guiding the detection of changed regions. Finally, a $k$-means is used to detect the 
truly changed regions. Though the approaches based on the combination of feature transform and clustering [14-16] provide better results than threshold segmentation-based approaches for change detection, these approaches are only suitable for images that have a clear difference between high frequency and low frequency, but unsuitable for many remote sensing images because of the complex background, texture, and illumination of images [17]. They usually fail to detect changed regions for images that have a complex background and blurred edges. Besides, these methods are sensitive to noise because fuzzy clustering algorithms are sensitive to noise [18,19].

The third group of approaches achieves change detection using region-based segmentation and the attributes of regions such as the ratio of length and width of objects, the shape of objects, the slope of objects, etc. The advantage of this kind of method is that it is insensitive to noise and is able to provide better contours. Zhang et al. [20] proposed a method of object-based change detection for high-resolution remote sensing images in urban areas. The method firstly employs region-based image segmentation to obtain the spatial attributes of objects. Then, the relationship between the spatial attributes of objects and characteristics of changed regions is explored to achieve change detection. However, the method proposed by Zhang et al. requires complex region descriptors to constrain changed regions. Ren et al. [21] compared pixel-based methods to region-based methods to demonstrate that the latter is able to provide better results since the region attribute is exploited and falsely-changed regions are removed. To overcome the problem, Lv et al. [22] proposed an object-based expectation maximization (OBEM) post-processing method to obtain truly-changed regions while removing falsely-changed regions. The method uses multi-scale segmentation to obtain region attributes and uses an expectation-maximization (EM) algorithm to compute truly-changed regions. Although change detection based on region segmentation has some advantages, the global information of images is missed, and manual feature descriptors are required [23]. More approaches used for change detection are reported in References [24-26].

Landslide mapping (LM) is an important research topic in change detection $[27,28]$. However, it is difficult to directly use change detection approaches to achieve LM due to the irregular texture, shape, and size of landslide regions. Therefore, the common approaches used for LM usually include two steps. First, change detection is used to obtain candidate landslide regions, i.e., coarse regions [8]. Second, an image post-processing approach is used to optimize the obtained landslide regions $[9,10]$. However, it is difficult to obtain effective candidate landslide regions [8]. Two difficulties in the problem need to be addressed. One difficulty is that the candidate landslide regions are obtained by computing the difference results of bitemporal images; the difference image only depends on the grayscale values of pixels but misses the structuring information of images. The other difficulty is that the existing approaches for LM have a clear high computational complexity for very-high resolution (VHR) remote sensing images.

To address the issues mentioned above, we propose a change detection approach based on fast FCM clustering (CDFFCM) and apply it to LM. The proposed method has two advantages:

(1) The difference image provided by the proposed CDFFCM is insensitive to illumination, noise, and environmental change. Furthermore, a large number of falsely changed regions are removed and truly changed regions are filled due to the incorporation of local spatial introduction and global image features.

(2) The proposed method has a low computational complexity because the Gaussian pyramid is able to remove the data redundancy of images for FCM. Moreover, the post-processing is fast because it is performed on binary images.

\section{Motivation}

According to the spectral characteristics of landslides, the grayscale value of pixels belonging to landslides is large. We can, therefore, obtain landslide regions using image segmentation. It is well known that threshold segmentation [29] is the simplest and the fastest approach for image segmentation. However, it is also the least effective because it is difficult to obtain a good segmentation 
result for the threshold segmentation. At present, a large number of intelligent segmentation algorithms, such as the active contour model [30], watershed [31], random walker [32], graph cut [33], spectral clustering [34], FCM [35], Gaussian mixed model [36], fully convolutional network (FCN) [37], etc., have been widely used in image segmentation. These algorithms can be categorized into four groups: partial differential equation (PDE)-based algorithms, region-based algorithms, graph-based algorithms, and pixel-based algorithms. Different algorithms are suitable for different types of image. As the computational complexity of most image segmentation algorithms is influenced by the image size, it is important to design a fast segmentation algorithm for VHR images.

Generally, PDE-based approaches [38] require prior information, i.e., an initial level set function, these approaches are noneffective for images with a complex background. Region-based approaches [39] usually rely on the quality of gradient images, but it is always difficult to obtain a good gradient image for remote sensing images due to noise and the complexity of the earth's surface. Graph-based approaches [40-42] usually rely on the construction of an affinity matrix. However, a high-resolution image usually corresponds to a huge similarity matrix, and the eigenvector decomposition of the matrix is time-consuming and memory-consuming. Although pixel-based segmentation approaches [43] are sensitive to noise, they are able to obtain more accurate segmentation results than the previous methods for high-resolution remote sensing images. In this paper, FCM is employed to obtain segmented bitemporal images that can be used for subsequent change detection.

\subsection{Motivation for Using Image Structuring Information}

Although a great number of change detection approaches $[6-8,11-13]$ have been proposed in the past decades, most of these approaches rely on the difference result obtained by bitemporal images and threshold segmentation. These approaches are thus complex and inefficient. Figure 1 shows the bitemporal images and their difference image.

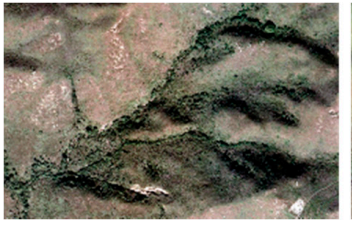

(a)

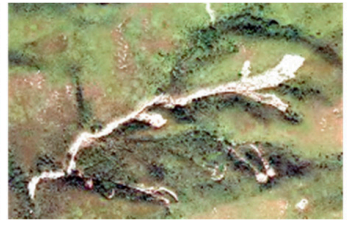

(b)

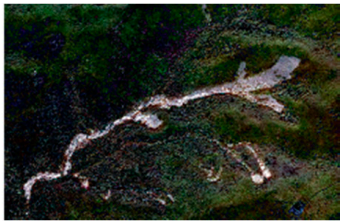

(c)

Figure 1. The bitemporal images and their difference image: (a) pre-event image at $t_{1},(\mathbf{b})$ post-event image at $t_{2}$, and (c) the difference image.

For Figure 1c, some popular approaches obtain changed regions using threshold segmentation. The segmentation result is sensitive to the threshold as shown in Figure 2.

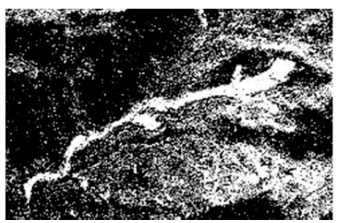

(a)

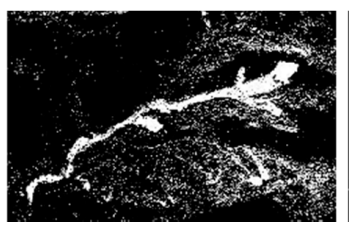

(b)

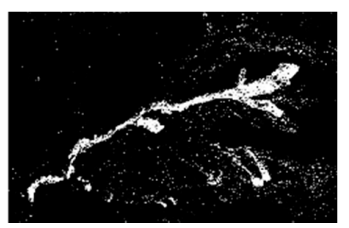

(c)

Figure 2. The difference images using single-threshold: (a) the threshold is 0.2 , (b) the threshold is 0.3 , and (c) the threshold is 0.4 .

To address the issue, Lv et al. [8] employ multi-thresholds and voting technologies to achieve change detection. Although the approach is superior to a single threshold, more parameters are necessary. Actually, it is a multi-threshold image segmentation approach by introducing local spatial neighboring information. Moreover, the approach is also sensitive to parameters. Therefore, it is 
unable to overcome the drawback of a single-threshold for change detection. To address the problem, we proposed a clustering-based image segmentation approach used for change detection. Although the difference image of bitemporal images is complex, as shown in Figure 1c, the structuring information of the bitemporal image is similar. We try to compute the difference image using the structuring information of images instead of the grayscale value. We have known that image segmentation is able to address the problem because the segmentation result of an image includes rich spatial structuring information that is useful for change detection.

To show our motivation for change detection, Figure 3 shows change detection based on the difference in grayscale value. It is clear that the difference image is sensitive to the change in grayscale value. To overcome the drawback, Figure 4 shows the effect of employing image structuring information for change detection.

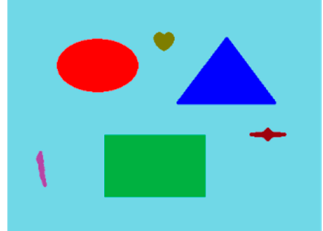

(a)

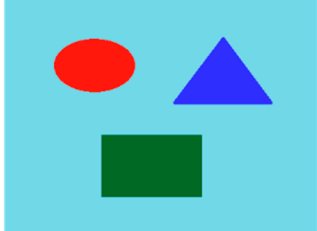

(b)

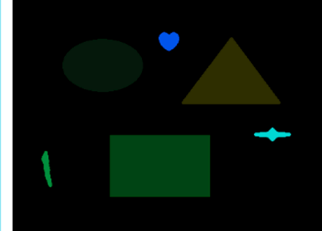

(c)

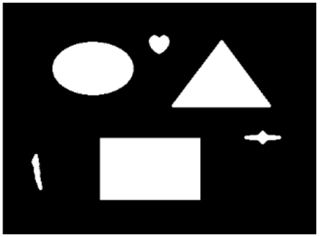

(d)

Figure 3. Change detection based on the difference in grayscale value: (a) image 1, (b) image 2, (c) the difference image, and (d) threshold segmentation (the threshold is 0.4).

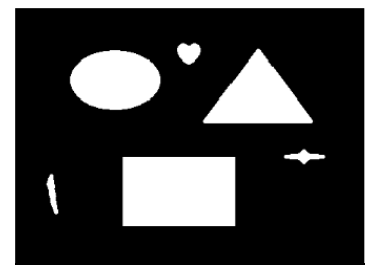

(a)

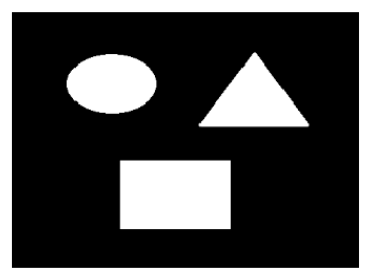

(b)

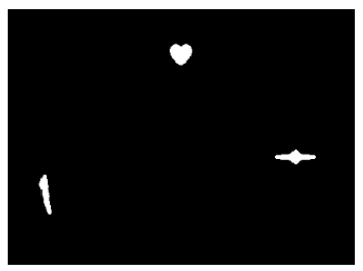

(c)

Figure 4. Change detection using the difference of image structuring information: (a) the structuring information corresponds to Figure $3 a$, (b) the structuring information corresponds to Figure $3 b$, and (c) the truly changed regions.

We can see that Figure $3 \mathrm{~d}$ includes falsely-changed regions because the difference image is sensitive to the change of grayscale values. Figure $4 \mathrm{c}$ shows a better result than Figure $3 \mathrm{~d}$ due to the utilization of the structuring information for the images in Figure 4c. Consequently, we can achieve a novel clustering-based image segmentation approach that is able to obtain accurately-changed regions by computing the structuring difference between bitemporal images.

\subsection{Motivation for Using a Gaussian Pyramid}

In this paper, FCM is first employed to generate segmentation results of bitemporal images. Second, the segmentation results are used for computing the difference of image structuring to obtain changed regions. Because the computational complexity of FCM is influenced by the image size, it requires a long running time to perform FCM on a VHR image. Although some improved FCM algorithms, such as Enhanced fuzzy c-means (EnFCM) [44] and fast and robust fuzzy c-means (FRFCM) [45], are able to reduce the computational complexity of FCM, they are only suitable for grayscale image. Both EnFCM and FRFCM employ a histogram to replace pixels to remove the redundant information of images. However, it is difficult to extend EnFCM and FRFCM to multi-band remote sensing images. The Gaussian pyramid [46] is an excellent approach for addressing the problem because it is able to remove the redundant information while preserving structuring information for multi-band images. We use the Gaussian pyramid to obtain multi-resolution images that have similar 
structuring information, and then implement FCM on a low-resolution image to speed up the algorithm. After that, we use the obtained clustering centers to compute the final membership on the original VHR image. Figure 5 shows the multi-resolution images obtained by the Gaussian pyramid.

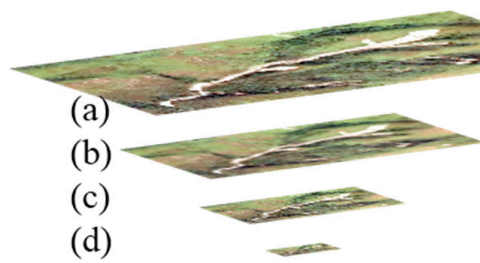

Figure 5. Multi-resolution images obtained using a Gaussian pyramid: (a) the original image of size $923 \times 593$, (b) the low-resolution image of size $461 \times 296$, (c) the low-resolution image of size $230 \times 148$, and (d) the low-resolution image of size $115 \times 74$.

Because FCM algorithms aim to obtain the clustering centers and classification labels, we can compute the clustering centers of a low-resolution image instead of the original high-resolution image from the Gaussian pyramid. Since there is a high data redundancy for images, we can obtain the similar clustering centers for multi-resolution images obtained by the Gaussian pyramid. Table 1 shows the clustering centers obtained by FCM for the image shown in Figure 5.

Table 1. Clustering centers of multi-resolution images shown in Figure 5 using $\operatorname{FCM}(c=5)$.

\begin{tabular}{ccccc}
\hline Clustering Center & (a) & (b) & (c) & (d) \\
\hline Center 1 & $57.4,43.6,26.5$ & $64.8,54.2,39.3$ & $72.8,65.3,52.2$ & $81.3,77.3,65.3$ \\
Center 2 & $113.5,110.2,87.6$ & $116.4,113.7,91.3$ & $118.4,116.3,93.6$ & $120.9,118.8,95.3$ \\
Center 3 & $149.9,147.8,111.4$ & $151.2,149.2,112.2$ & $152.4,150.4,112.7$ & $154.4,152.4,113.9$ \\
Center 4 & $173.7,179.5,130.6$ & $172.9,178.7,128.9$ & $172.1,178.0,127.4$ & $154.4,152.4,113.9$ \\
Center 5 & $245.8,246.3,240.5$ & $243.0,242.1,235.1$ & $239.9,237.8,229.5$ & $235.6,232.3,222.2$ \\
\hline
\end{tabular}

Furthermore, we can obtain the segmentation results of original bitemporal images using clustering centers shown in Table 1. Figure 6 shows the corresponding segmentation results, where five colors represent five classes.

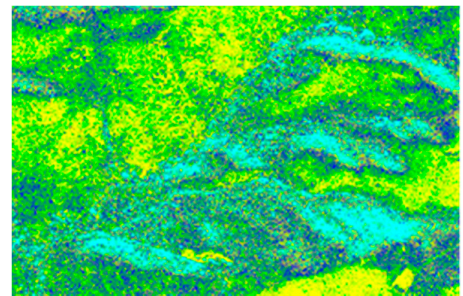

(a)

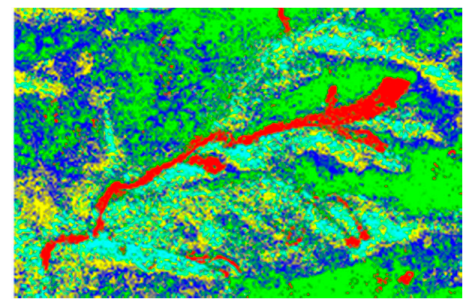

(b)

Figure 6. Segmentation results of pre- and post-event images shown in Figure 5 using FCM $(c=5)$ : (a) pre-event image, and (b) post-event image.

In Table 1, we can see that the clustering centers are similar for four different-resolution images shown in Figure 5. Therefore, a fast clustering algorithm can be achieved by using Gaussian pyramid for VHR images.

\section{Methodology}

The framework of the proposed CDFFCM used for LM is shown in Figure 7. It consisted of the following steps: 
(1) Input bitemporal images and set parameters: $T_{1}$ is a threshold used for comparing the values of clustering centers, and it was used for the image binarization as well. $c$ is the number of clusters used for FCM.

(2) Fast image segmentation: A VHR image included a large number of pixels, which meant that it was time-consuming to achieve image segmentation for the VHR image. We employed the Gaussian pyramid to obtain low-resolution images that were applied to FCM to reduce the computational burden for the VHR images.

(3) Change detection using difference of image structuring information: Because the pre-event and post-event images were segmented by FCM, respectively, we could compute the difference image according to the segmented images without using original bitemporal images.

- The structuring information of the post-event image was extracted, i.e., the candidate landslide regions were extracted from the post-event image according to the maximal module value of its clustering centers.

- The structuring information of the pre-event image was extracted, i.e., the false landslide regions were extracted from the pre-event image according to the maximal module value of its clustering centers or the threshold $T_{1}$.

- The difference of structuring information was obtained, i.e., the changed regions were extracted by computing the difference between the candidate landslide regions and the false landslide regions.

(4) Post-processing: Because the difference image was coarse and included lots of false landslide regions, morphological filtering and filling technologies were applied to the modification of LM. Furthermore, we employed a morphological reconstruction to remove false landslide regions to improve the accuracy of LM.

(5) Output result:

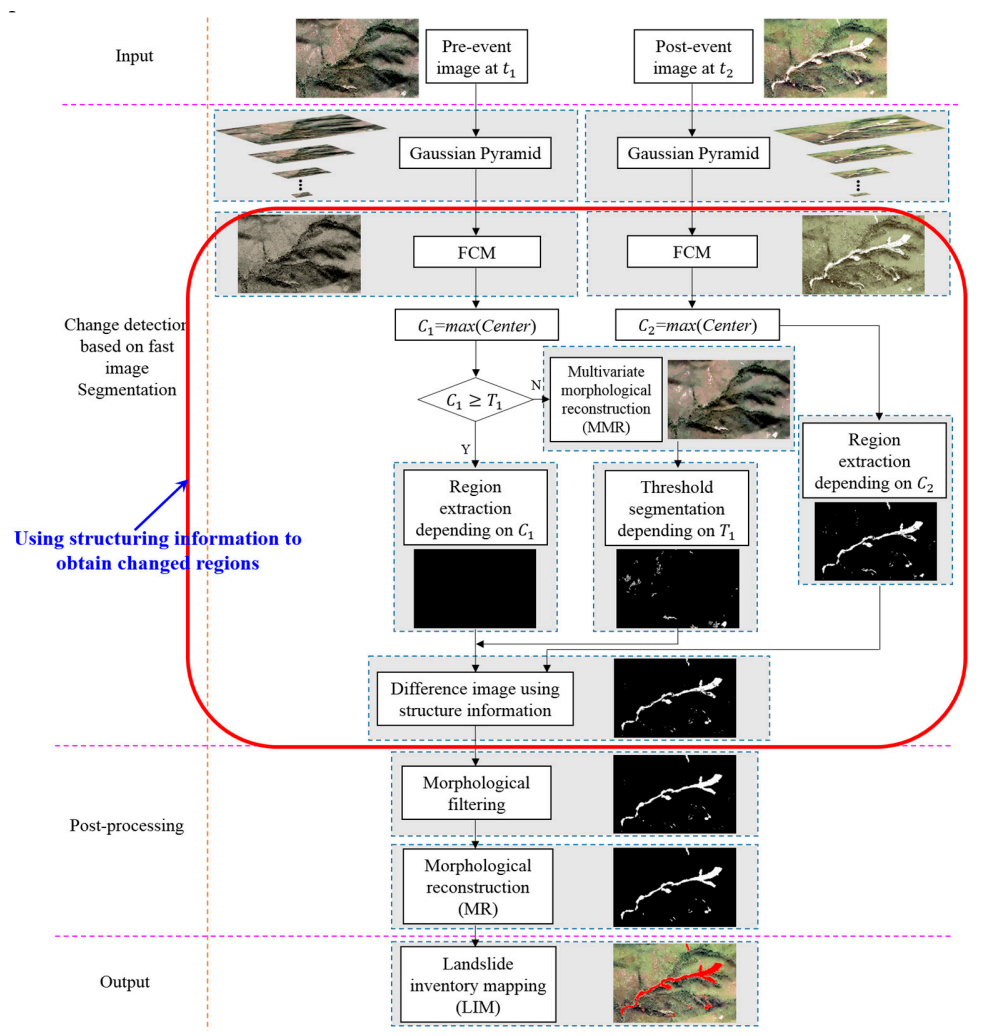

Figure 7. The framework of CDFFCM for landslide mapping (LM). 


\subsection{Fast FCM for Change Detection}

In this paper, we employed a pixel-based clustering algorithm to achieve image segmentation. Because FCM is one of the most popular clustering algorithms, we applied FCM to bitemporal images to obtain changed regions; the objective function of FCM algorithms is defined as follows:

$$
J_{m}=\sum_{i=1}^{N} \sum_{k=1}^{c} u_{k i}^{m}|| x_{i}-v_{k} \|^{2},
$$

where $x_{i}$ is the gray value of the $i$ th pixel, $v_{k}$ represents the prototype value of the $k$ th cluster, and $u_{k i}$ denotes the fuzzy membership value of the $i$ th pixel with respect to cluster $k . N$ is the total number of pixels in an image $f$, and $c$ is the number of clusters. The parameter $m$ is a weighting exponent on each fuzzy membership that determines the amount of fuzziness of the resulting classification.

According to the definition of $J_{m}$ and the constraint that $\sum_{k=1}^{c} u_{k i}=1$ for each pixel $x_{i}$, and using the Lagrange multiplier method, the calculation of the membership partition matrix and the clustering centers is given as follows:

$$
\begin{gathered}
u_{k i}=\frac{\left\|x_{i}-v_{k}\right\|^{-2 /(m-1)}}{\sum_{j=1}^{c}\left\|x_{i}-v_{j}\right\|^{-2 /(m-1)}}, \\
v_{k}=\sum_{i=1}^{N} u_{k i}^{m} x_{i} / \sum_{i=1}^{N} u_{k i}^{m} .
\end{gathered}
$$

It can be seen from Equations (1)-(3) that FCM has two drawbacks for image segmentation. One is that FCM is sensitive to noise due to the missing of spatial information of an image. The other one is that the computational complexity of FCM is influenced by the number of pixels in the image. To address these drawbacks, the local spatial information is often incorporated into the objective function of FCM [47-49]. Although the introduction of local spatial information is able to reduce the influence of noise for image segmentation, it also leads to a high computation complexity, especially for high-resolution images. The objective function of the improved FCM algorithms is defined as:

$$
J_{m}=\sum_{i=1}^{N} \sum_{k=1}^{c} u_{k i}^{m}|| x_{i}-v_{k} \|^{2}+\sum_{i=1}^{N} \sum_{k=1}^{c} u_{k i}^{m} G_{k i}
$$

where $G_{k i}$ represents fuzzy factor; it is used to control the influence of neighborhood pixels on the central pixel, whereas different $G_{k i}$ usually leads to a variant clustering algorithm. Similar to Equations (2) and (3), the membership and clustering centers of improved FCM algorithms are obtained as follows:

$$
\begin{gathered}
u_{k i}=\frac{\left(\left\|x_{i}-v_{k}\right\|^{-2}+G_{k i}\right)^{-1 /(m-1)}}{\sum_{j=1}^{c}\left(\left\|x_{i}-v_{j}\right\|^{-2}+G_{j i}\right)^{-1 /(m-1)}}, \\
v_{k}=\sum_{i=1}^{N} u_{k i}^{m} x_{i} / \sum_{i=1}^{N} u_{k i}^{m} .
\end{gathered}
$$

The improved fuzzy clustering algorithm has a clearly high computational complexity for VHR images. According to Equations (1) and (4), the computational complexity of the FCM algorithm is influenced by the values of $N$ and $c$. The execution time of the FCM algorithm will be longer when the image size larger or $c$ is larger. Thus, we can reduce the computational complexity of FCM by removing the redundant information of images while preserving the structuring information of images.

Inspired by the FRFCM and the Gaussian pyramid, the Gaussian pyramid is first used to generate multi-resolution images and FCM is then performed on a low-resolution image to achieve fast image segmentation. After that, membership filtering is performed on labels. As a result, the candidate landslide regions are obtained. Figure 8 shows candidate landslides for the image shown in Figure $1 \mathrm{~b}$ using the proposed fast FCM algorithm. Table 2 shows the comparison of execution time and iteration times for multi-resolution images shown in Figure 5. 


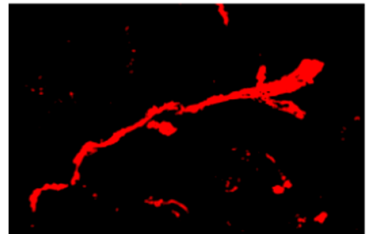

(a)

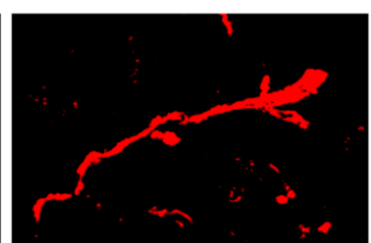

(b)

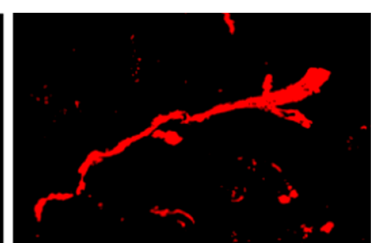

(c)

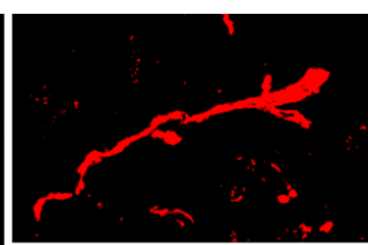

(d)

Figure 8. Candidate landslides of Figure $1 \mathrm{~b}$ using four groups of clustering centers in Table 1: (a) candidate landslide using clustering centers shown in Table 1a, (b) candidate landslide using clustering centers shown in Table 1b, (c) candidate landslide using clustering centers shown in Table 1c, and (d) candidate landslide using clustering centers shown in Table $1 \mathrm{~d}$.

Table 2. Execution time (in seconds) of the FCM algorithm on multi-resolution images shown in Figure $5(c=5)$.

\begin{tabular}{ccccc}
\hline & (a) & (b) & (c) & (d) \\
\hline Iterations & 100 & 88 & 66 & 62 \\
Time & 69.84 & 17.48 & 3.28 & 0.98 \\
\hline
\end{tabular}

The results shown in Figure 8 are similar because of the similar clustering center values. The regions marked by red color correspond to clustering center 5, i.e., candidate landslides. Table 2 shows that a low-resolution image requires shorter execution time to compute clustering centers. Figure 8 and Table 2 show that the proposed fast FCM algorithm obtains accurate segmentation results while reducing the execution time of the FCM algorithm. In practical applications, the selection of a low-resolution image provided by the Gaussian pyramid is decided by the image resolution of bitemporal images.

\subsection{Post-Processing for Change Detection}

Although changed regions can be obtained by computing the difference results of segmented bitemporal images, there are still a lot of falsely-changed regions caused by noise and illumination variation. Figure 9 shows candidate landslides of the bitemporal images where the landslide regions have a high brightness in Figure 9c. Therefore, it was easy to obtain candidate landslides from the segmentation result according to clustering centers. The candidate landslides are shown in Figure $9 \mathrm{~d}$. However, many false landslide regions were also detected. It was necessary to remove false landslide regions by using change detection based on the image structuring information that is defined as:

$$
\begin{gathered}
L_{x y}^{d}=L_{x y}^{p o s t}-L_{x y}^{p r e}, \\
L_{x y}^{d b}=\left\{\begin{array}{ll}
1, & L_{x y}^{d}>0 \\
0, & L_{x y}^{d} \leq 0
\end{array},\right.
\end{gathered}
$$

where $x$ and $y$ are the corresponding column and row, respectively, in the bitemporal images and difference image. $L^{\text {pre }}$ and $L^{\text {post }}$ represent candidate landslide regions of pre-event and post-event images, respectively. $L^{d}$ and $L^{d b}$ denote the difference image and binarized difference image, respectively.

We apply Equations (7) and (8) to two groups of bitemporal images to detect changed regions. Figure $9 \mathrm{~b}$ shows all the false landslide regions that were removed completely because these regions were too small. Therefore, we could not remove false landslide regions in Figure $9 \mathrm{~d}$ according to Equations (7) and (8). On the contrary, Figure 10 shows false landslide regions could be removed by using Equations (7) and (8). 
By comparing Figure 9 to Figure 10, we find that there were two cases for the removal of false landslide regions using Equations (7) and (8). In the first case, we could remove false landslide regions when the maximal value of clustering centers of the pre-event images was larger than $T_{1}$, e.g., Figure 10. It was the opposite in the second case, where we could not remove false landslide regions under the second case, i.e., the maximal value of clustering centers of pre-event image was smaller than $T_{1}$, e.g., Figure 9. To address the problem of the removal of false landslide regions, we employed a multivariate morphological reconstruction (MMR) [50] to process the pre-event image and obtain false landslide regions using the threshold $T_{1}$ for the second case. The reconstruction and segmentation results of the pre-event image are shown in Figure 11.

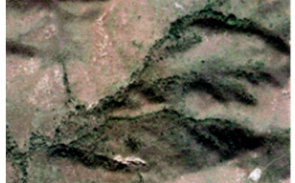

(a)

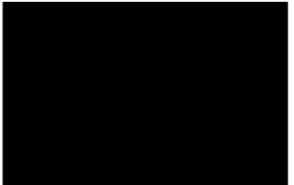

(b)

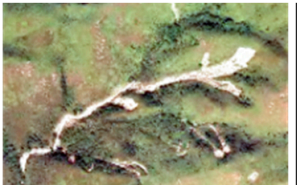

(c)

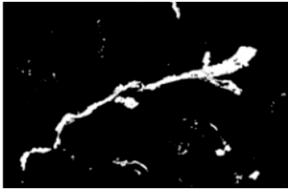

(d)

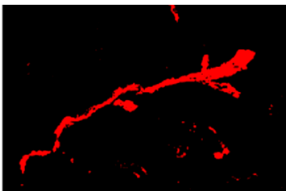

(e)

Figure 9. Landslide mapping using change detection based on image structuring information $\left(T_{1}=0.8\right.$, the grayscale value of the image is normalized from 0 to 1): (a) pre-event image; (b) candidate false landslide regions $L^{\text {pre }}$; (c) post-event image; (d) candidate landslide regions $L^{\text {post }}$; and (e) detected landslide regions according to $L^{\text {pre }}$ and $L^{\text {post }}$, where regions marked by red color represent true landslide regions and regions marked by cyan color represent removed false landslide regions.

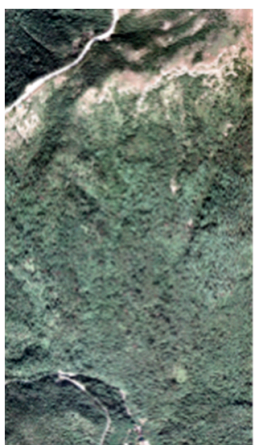

(a)

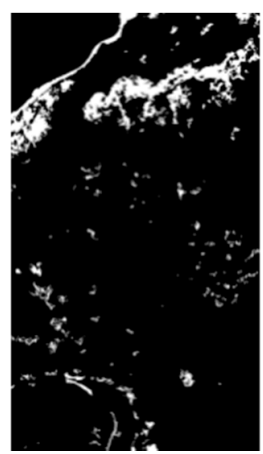

(b)

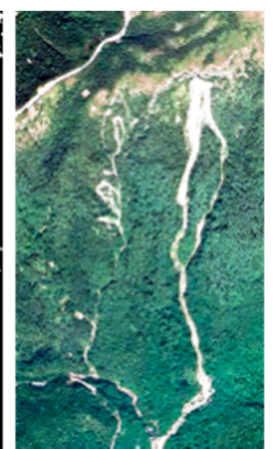

(c)

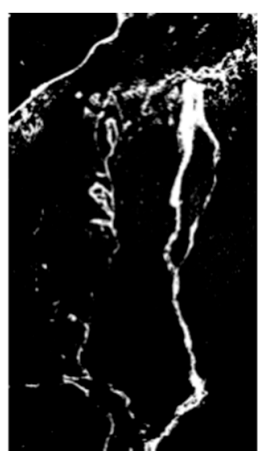

(d)

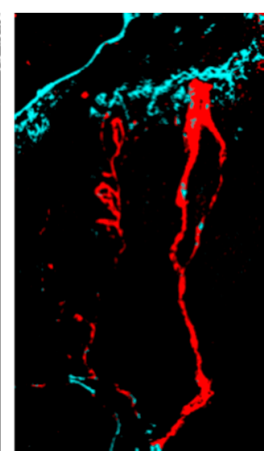

(e)

Figure 10. Landslide mapping using change detection based on image structuring information $\left(T_{1}=0.8\right.$, the grayscale value of the image is normalized from 0 to 1 ): (a) pre-event image; (b) candidate false landslide regions $L^{\text {pre }}$; (c) post-event image; (d) candidate landslide regions $L^{\text {post }}$; and (e) detected landslide regions according to $L^{\text {pre }}$ and $L^{\text {post }}$, where regions marked by red color represent true landslide regions and regions marked by cyan color represent removed false landslide regions.

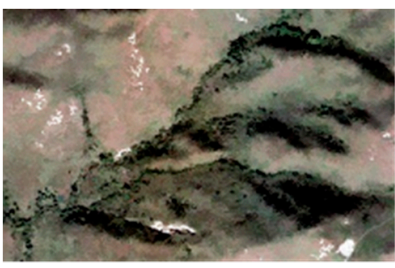

(a)

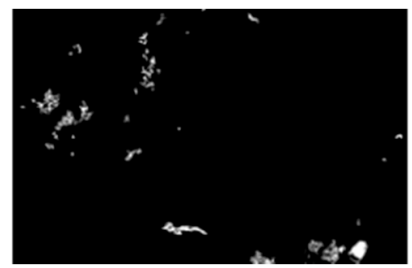

(b)

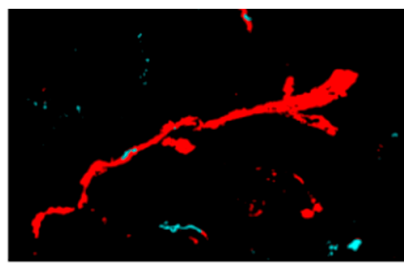

(c)

Figure 11. Reconstruction and segmentation results of the pre-event image: (a) reconstruction result using MMR (the structuring element is a disk of size $5 \times 5$ ); (b) candidate false landslide regions $L^{\text {pre }}$ using the threshold method, $T_{1}=0.8$; and (c) detected landslide regions according to the difference between Figures $9 \mathrm{~d}$ and $11 \mathrm{~b}$, where regions marked by red color represent true landslide regions and regions marked by cyan color represent removed false landslide regions. 
Figure 11 shows that the proposed approach overcame the problem existing in the second case, i.e., false landslide regions were removed from the candidate landslide regions. Based on the analysis above, the true landslide regions were obtained easily by Equations (7) and (8). Figures 10e and 11c show that true landslide regions were detected and false landslide regions were removed by the proposed approach. Therefore, it was easier to achieve landslide mapping using the difference of the image structuring information instead of the difference of the grayscale value. However, there were still a lot of false landslide regions. We could employ morphological filters and morphological reconstruction operations to remove these false landslide regions.

Because the detected landslide regions included many holes in large regions and many isolated points, we needed to fill holes while removing isolated points. We designed an effective morphological filter that was able to remove isolated points while filling holes.

Let $S$ denote a set whose elements are eight-connected boundaries, each boundary enclosing a background region (i.e., a hole). Based on the basic morphological dilation and complementation operations, the operation of filling holes can be defined as follows:

$$
X_{m}=\delta\left(X_{m-1}, b\right) \cap S^{c},
$$

where $m \in N^{+}, X_{0}$ is an initial array (the same size as the array containing $S$ ), $b$ is a structuring element that is a disk of size $3 \times 3$, and $\delta$ denotes the morphological dilation operation. All the holes will be filled until $X_{m}=X_{m-1}$. The final output denoted by $S^{\prime}$ is defined as:

$$
S^{\prime}=X_{m} \cup S
$$

If we use the symbol $F$ to denote the operation of filling holes, we propose an effective morphological filtering method that is suitable for smoothing landslide regions,

$$
S^{\prime \prime}=\varepsilon(F(\delta(S, B)), B),
$$

where $B$ is a structuring element that is used for connecting leaking regions. Generally, the size of $B$ is larger with the increase of the image size. Let $r$ be the radius of $B$, then

$$
r=\operatorname{round}(\min (W, H) / 500)+1,
$$

where $W$ and $H$ denote the width and height of bitemporal images, respectively. Because the size of $B$ is decided by the image size, the proposed morphological filter is free parameters. It can be seen from Equation (11) that the proposed filtering method is able to fill holes due to $F$ and the closing operation. However, the method was unable to remove small regions caused by noise or false landslides. Because morphological reconstruction (MR) [51] was able to remove small regions while maintaining the large regions in an image, we could employ MR to remove false landslide regions. The reconstructed result of the image was often decided by the size of the structuring element. Generally, the larger structuring element, the more small regions that are removed for morphological dilation reconstruction. The basic morphological erosion and dilation reconstructions are defined as:

$$
\left\{\begin{array}{ll}
R^{\varepsilon}(f, B)=\varepsilon_{f}^{(n)}(g) \vee f, & g=\delta(f, B) \\
R^{\delta}(f, B)=\delta_{f}^{(n)}(g) \wedge f, & g=\varepsilon(f, B)
\end{array},\right.
$$

where $g$ is a marker image that $g \geq f$ for $R^{\varepsilon}$ and $g \leq f$ for $R^{\delta}$. B is the structuring element used in both Equations (11) and (13), where $\varepsilon$ denotes a morphological erosion operation. $R^{\varepsilon}$ and $R^{\delta}$ denote morphological erosion and dilation reconstruction, respectively. Based on $R^{\varepsilon}$ and $R^{\delta}$, the combinational morphological reconstruction operations that are more popular in practical 
applications, i.e., morphological opening and closing reconstructions denoted by $R^{O}$ and $R^{C}$, are proposed as follows:

$$
\left\{\begin{array}{l}
R^{O}(f, B)=R^{\delta}\left(R^{\varepsilon}(f, B), B\right) \\
R^{C}(f, B)=R^{\varepsilon}\left(R^{\delta}(f, B), B\right)
\end{array} .\right.
$$

It can be seen from Equation (13) that morphological dilation reconstruction is able to remove objects that are smaller than $B$ while morphological erosion reconstruction has the opposite effect due to duality. Therefore, we employ $R^{C}$ shown in Equation (14) to optimize the detected landslides $S^{\prime \prime}$.

\section{Experiments}

In order to evaluate the proposed approach, three groups of bitemporal VHR landslide remote sensing images were used in our experiments. Three popular approaches $[9,10]$, i.e., edge-based level-set (ELSE), region-based level-set (RLSE) [10], and change detection-based Markov random field (CDMRF) [9] were considered as comparative approaches to demonstrate the effectiveness and efficiency of CDFFCM for LM. All approaches were implemented with MATLAB 2017b (https:/ / www.mathworks.com/) and performed on a DELL desktop with Intel(R) Core(TM) CPU, i7-6700, 3.4 GHz, and 16 GB RAM (Dell, Shanghai, China).

\subsection{Data Description}

Three groups of bitemporal images on A-area, B-area, and C-area were captured by the Zeiss RMK TOP 15 Aerial Survey Camera System (Jena, Germany) at a flying height of approximately $2.4 \mathrm{~km}$ in December 2007 and in November 2014, respectively. The locations of A-area, B-area, and C-area are shown in Figure 12. The geometrical resolution of the bitemporal images is $0.5 \mathrm{~m}$. All images used in the experiments were preprocessed via geometry registration and radiometric correction.

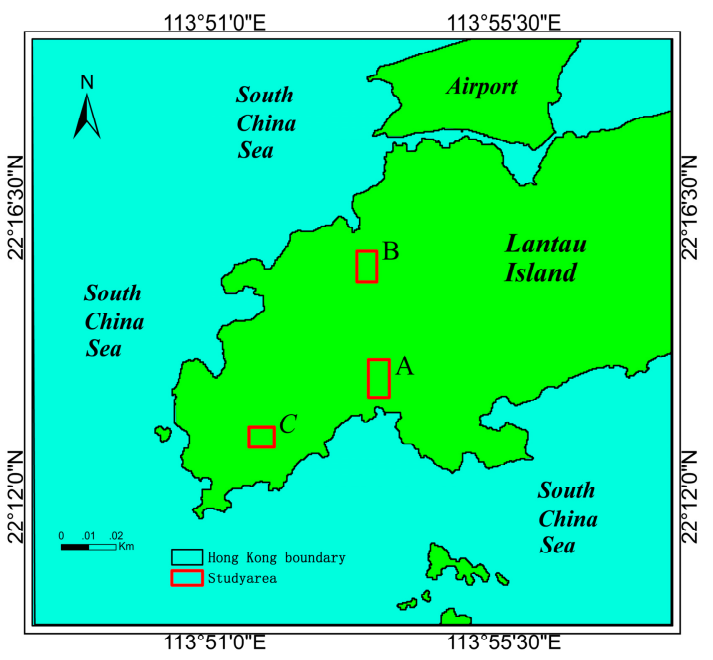

Figure 12. Study area locations of A-area, B-area, and C-area on Lantau Island, Hong Kong, China.

- A-area: Figure 13a,b shows the pre- and post-event images of A-area. The size of the bitemporal images was $1252 \times 2199$. The true boundaries of the landslides in the images were generated manually by human experts in the field of remote sensing, and they are shown in Figure 13c. Figure $13 \mathrm{~d}$ shows the corresponding ground truth segmentation.

- B-area: Figure 14a,b shows the pre- and post-event images of B-area. The size of the images was $923 \times 593$. The ground truths, i.e., the boundaries and segmentation results of landslides in the post-event image, are shown in Figure 14c,d, respectively. 
- C-area: Figure 15a,b shows the pre- and post-event images of C-area. The size of the images was $750 \times 950$. The ground truths, i.e., the boundaries and segmentation results of landslides in the post-event image are shown in Figure 15c,d, respectively.

\subsection{Experimental Setup}

To fairly compare the performance of different approaches, the parameter values used for the experiments follow the original papers. The parameter values, $\alpha=1.5, C_{0}=1.0$, and $\sigma_{1}=\sigma_{2}=1.0$ were used for ELSE and RLSE, where $\alpha$ is a constant coefficient to reduce the effect of data dispersion on the experimental results by increasing the standard deviation term, and $\sigma$ is the standard deviation of the Gaussian filter used for preprocessing. The template size of the Gaussian filter was $9 \times 9$, and the time step was $\Delta T=5.0$. The parameter values used for CDMRF were as follows: $T=1.0, \Delta T=1.5, \lambda=50$, and $M=5$. The value of $(T+\Delta T)$ was consistent with the effect of $\alpha$, i.e., it aimed to reduce the effect of data dispersion on the experimental results. $\lambda$ is a weighting coefficient which was used to balance the pairwise potential. $M$ is the number of clusters. To our knowledge, the value of the parameters were the best for the comparative approaches. For the proposed CDFFCM, only two parameters, $T_{1}$ and $c$ were required, and other parameters could be computed automatically according to the size of the bitemporal images. We set $T_{1}=0.8$ and $c=5$ in this experiment. Both the structuring elements used for image filtering and morphological reconstruction, and the multiple of downsampling used for a Gaussian pyramid, were decided by the size of the bitemporal images.

In order to quantitatively compare CDMRF, ELSE, and RLSE with the proposed CDFFCM, four quantitative evaluation indices [10] are presented:

$$
\begin{gathered}
\text { Precision }=P_{l m} / P_{l}, \\
\text { Rcall }=P_{l m} / P_{r}, \\
\text { F-score }=2 \times \frac{\text { Precision } \times \text { Rcall }}{\text { Precision }+ \text { Rcall }}, \\
\text { Accuracy }=P_{l m} /\left(P_{l}+P_{\text {rum }}\right),
\end{gathered}
$$

where $P_{l m}$ is the total pixel number of the identified landslides that are matched with the corresponding ground truth, $P_{r}$ is the total pixel number of the ground truth, $P_{l}$ is the total pixel number of the identified landslides, and $P_{\text {rum }}$ is the total pixel number of the corresponding ground truth that is not matched with the identified landslides. $P_{\text {over }}$ is the total pixel number of detected false landslides.

\subsection{Experimental Results}

\subsubsection{A-Area}

Figure 13a,b show the pre-event and post-event images for A-area, respectively. The landslide regions are clear in Figure 13b. Although we could recognize landslide regions visually according to the difference between Figure 13a,b, it was difficult to achieve the computer recognition due to a complex background and noise. There was also a clear difference between Figure 13a,b for background regions due to the influence of the illumination. Figure $13 \mathrm{c}$ shows the contour of true landslide regions that was delineated by experts in the field of remote sensing, and Figure 13d shows the binary image of landslide regions corresponding to Figure 13c. Figure 13e-h shows the identification results of landslide regions generated by ELSE, RLSE, CDMRF, and the proposed CDFFCM, respectively. Compared to the first three results, the last one provided a better visual effect for LM. Moreover, the last one was closer to the ground truth than the other results. The first three results included a great number of breakage regions due to missed detections. Because the bitemporal images were obtained in December 2007 and in November 2014, respectively, the landslide regions had changed during the seven years due to environmental change. Figure $13 \mathrm{~b}$ shows that some landslide regions were covered by trees, grass, or soil. Besides, two images taken at different times on the same unchanged 
area were also different due to the influence of the imaging system, environment, and noise. Therefore, the difference result of bitemporal images usually included a large number of falsely-changed regions caused by illumination or noise. Meanwhile, the difference image usually missed a lot of truly changed regions as well due to fresh trees or grass on landslide regions.

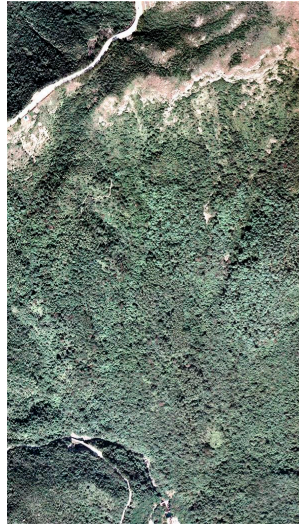

(a)

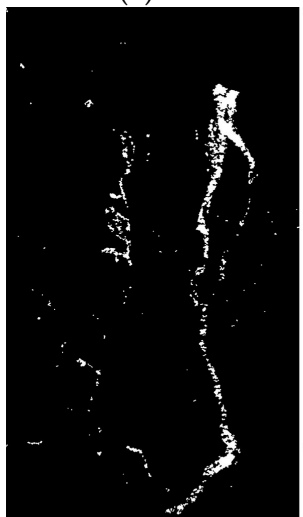

(e)

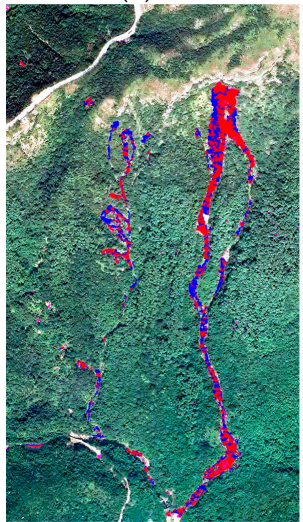

(i)

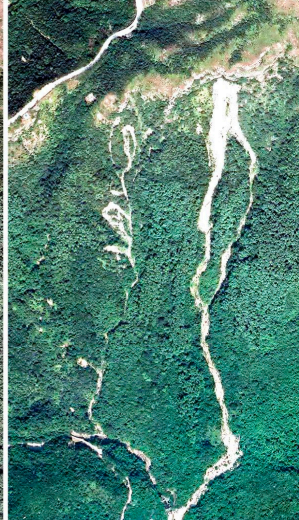

(b)

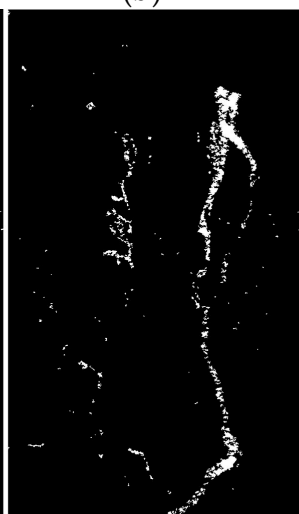

(f)

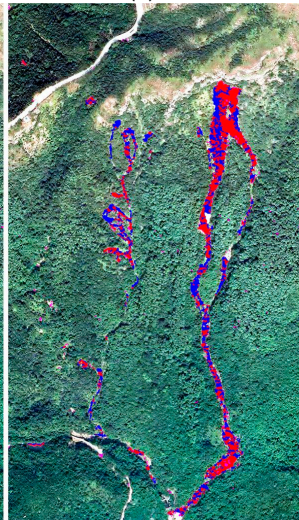

(j)

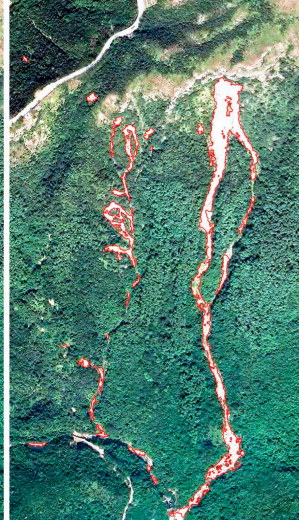

(c)

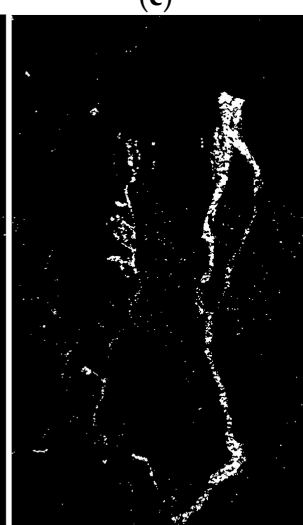

(g)

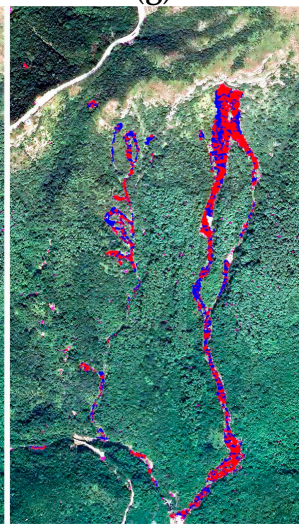

(k)

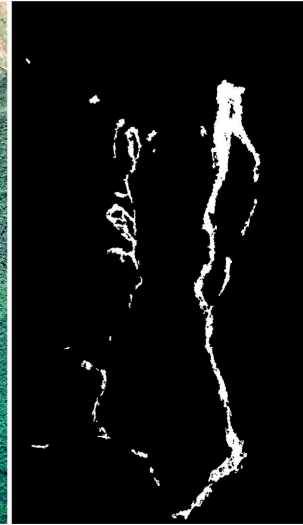

(d)

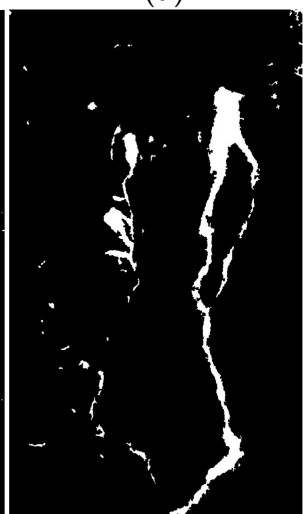

(h)

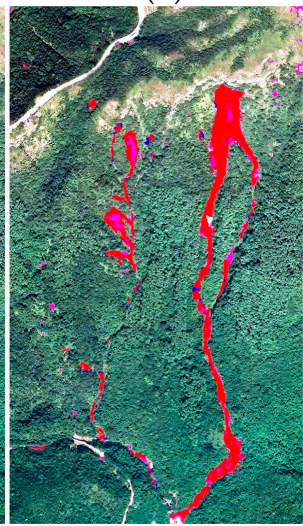

(1)

Figure 13. Experimental results on A-area: (a) pre-event image; (b) post-event image; (c) contour of ground truth on post-event image; (d) ground truth segmentation; (e) landslide regions obtained by ELSE; (f) landslide regions obtained by RLSE; (g) landslide regions obtained by CDMRF; (h) landslide regions obtained by the proposed CDFFCM; and (i-l) landslide mapping results using ELSE, RLSE, CDMRF, and CDFFCM overlaid on the post-event image, respectively.

As traditional change detection approaches, such as ELSE, RLSE, and CDMRF, rely on the difference result of bitemporal images, the final identification results had a clear difference from the ground truth, as shown in Figure 13e-g. Compared to ELSE, both RLSE and CDMRF provided a better visual effect 
due to two reasons. One is that RLSE employs region segmentation instead of the gradient segmentation employed by ELSE. The other reason is that CDMRF had a stronger capability of noise suppression due to the introduction of spatial context information of landslides. Figure $13 \mathrm{~h}$ shows the result obtained by the proposed CDFFCM that overcame the shortcomings existing in ELSE, RLSE, and CDMRF. Because the proposed CDFFCM employed fuzzy clustering to obtain the spatial structuring information of bitemporal images, the difference result relied on the structuring change other than the change of grayscale values. The proposed approach was therefore insensitive to noise. It was not only able to exclude falsely-changed regions, but was also able to supply the missed landslide regions that were truly changed. Figure 13i-1 shows the comparative results of landslide mapping, where the regions marked by red color were true-detected landslide regions, the regions marked by pink were false-detected landslide regions, and the regions marked by blue were true-missed landslide regions. According to Figure 131, the proposed CDFFCM provided the best result for LM because the area of red regions was the largest while the sum of pink and blue regions was small.

\subsubsection{B-Area}

Figure 14a,b shows the pre-event and post-event image for B-area, respectively. The landslide regions shown in Figure $14 \mathrm{~b}$ were clearer than those in A-area. There was a very clear difference between Figure 14a,b due to the environmental change. Figure 14c shows the contour of true landslide regions and Figure $14 \mathrm{~d}$ shows the landslide regions corresponding to Figure $14 \mathrm{c}$. We can see that there was less bare rock or soil in Figure 14a compared to Figure 14b. Figure 14e-h shows the identification results of landslide regions generated by ELSE, RLSE, CDMRF, and the proposed CDFFCM. Similar to Figure 13, the last one generated by the proposed CDFFCM provided a better visual effect than the first three results for LM. Moreover, the last one was closer to the ground truth than the three previous results. The four approaches used for LM generated better results for Figure 14 than Figure 13 since B-area included less noise. Figure 14i-1 shows the comparison result of different approaches for landslide mapping, where the result generated by the proposed CDFFCM included more red regions and less blue regions in Figure 141.

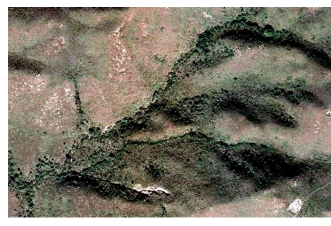

(a)

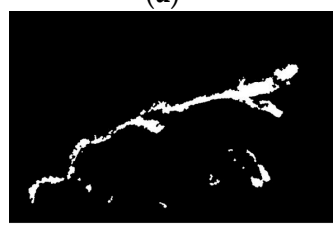

(e)

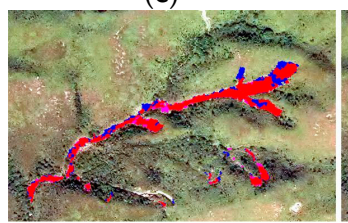

(i)

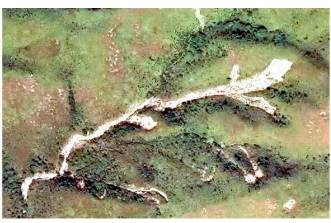

(b)

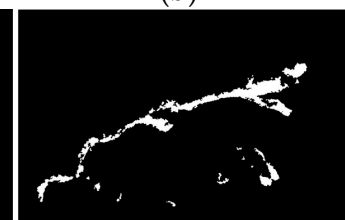

(f)

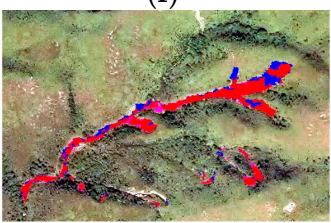

(j)

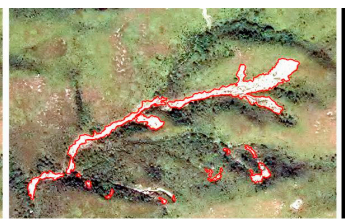

(c)

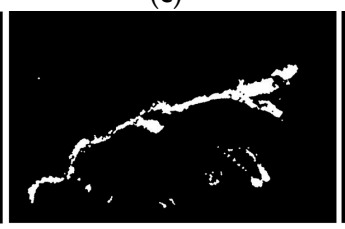

(g)

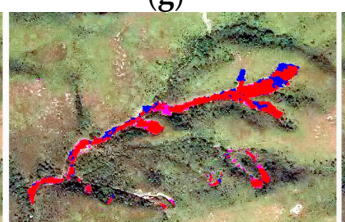

(k)

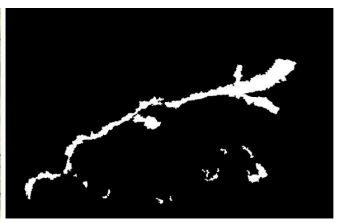

(d)

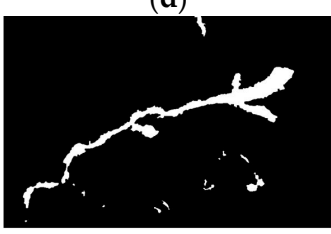

(h)

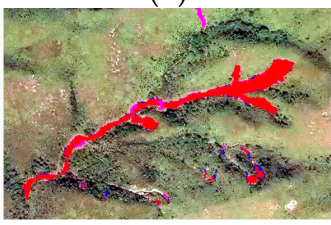

(1)

Figure 14. Experimental results on B-area. (a) pre-event image; (b) post-event image; (c) contour of ground truth on post-event image; (d) ground truth segmentation; (e) landslide regions obtained by ELSE; (f) landslide regions obtained by RLSE; (g) landslide regions obtained by CDMRF; (h) landslide regions obtained by the proposed CDFFCM; and (i-l) landslide mapping results using ELSE, RLSE, CDMRF, and CDFFCM overlaid on the post-event image, respectively. 
Although A-area is different from B-area in image size and texture features, the proposed CDFFCM achieved the best identification results in four approaches for both A-area and B-area. Furthermore, the proposed CDFFCM had a stronger robustness for LM.

\subsubsection{C-Area}

Compared to A-area and B-area, it was more difficult to obtain accurate landslide regions for C-area due to the complexity of landslides. Figure 15a,b shows the pre-event and post-event image for $\mathrm{C}$-area, respectively. We can see that some landslide regions in Figure $15 \mathrm{~b}$ were covered by the fresh vegetation of the surrounding. Therefore, it was more difficult and took more time to delineate the true landslide regions in C-area than for A-area and B-area. Figure 15c shows the contour of true landslide regions of the post-event and Figure 15d shows the landslide regions corresponding to Figure 15c. Figure $15 \mathrm{e}-\mathrm{h}$ shows the identification results of landslide regions generated by ELSE, RLSE, CDMRF, and the proposed CDFFCM. The proposed CDFFCM provided the best result since Figure 15h was closer to the ground truth shown in Figure 15d than Figure 15e-g.

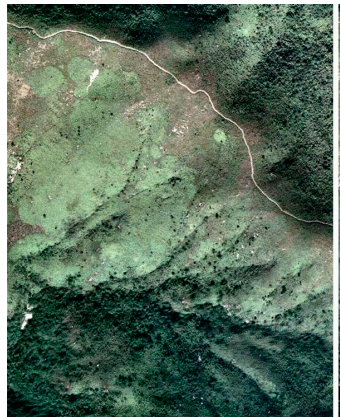

(a)

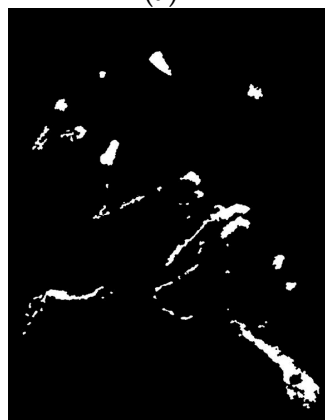

(e)

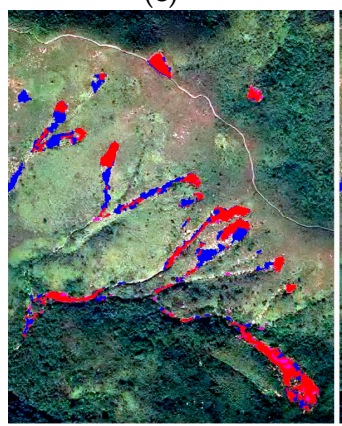

(i)

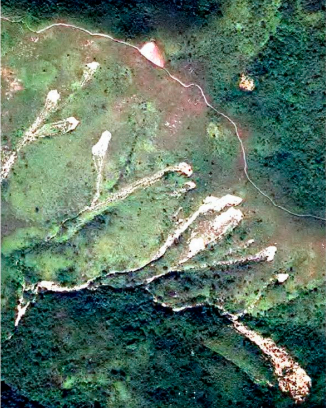

(b)

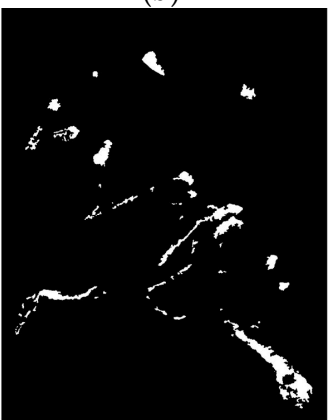

(f)

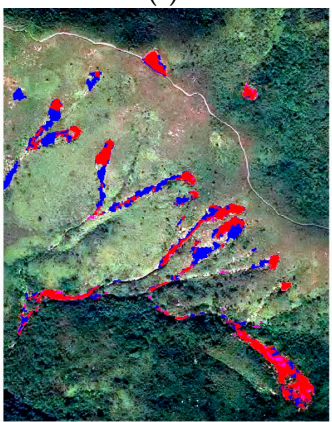

(j)

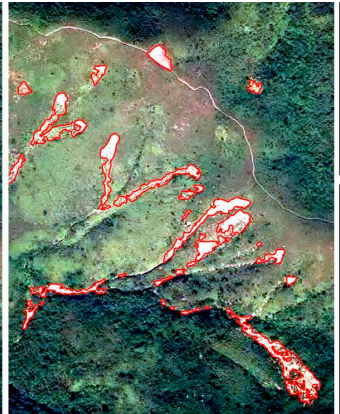

(c)

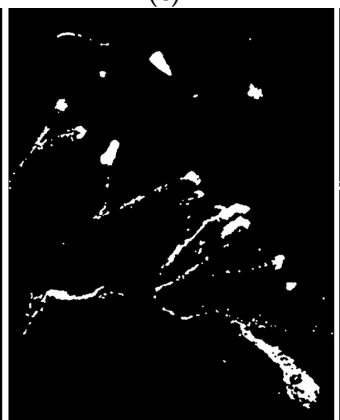

(g)

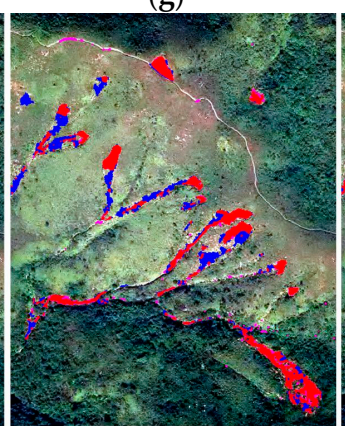

(k)

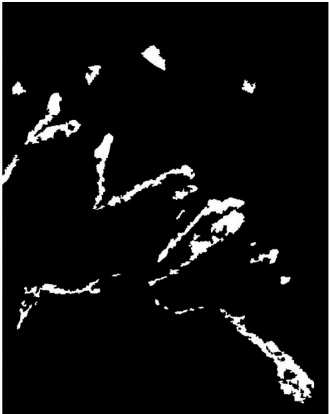

(d)

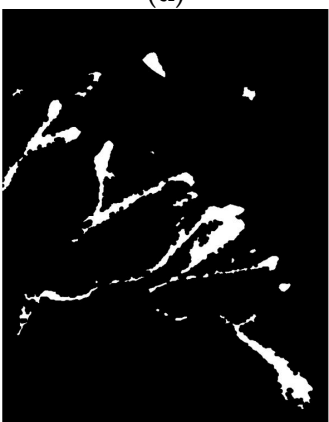

(h)

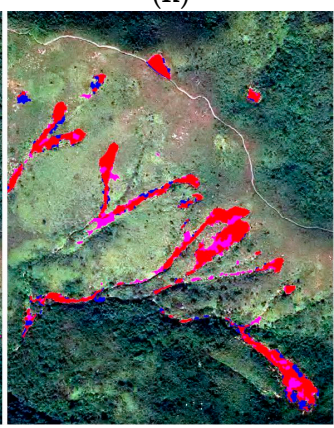

(1)

Figure 15. Experimental results on C-area: (a) pre-event image; (b) post-event image; (c) contour of ground truth on post-event image; (d) ground truth segmentation; (e) landslide regions obtained by ELSE; (f) landslide regions obtained by RLSE; (g) landslide regions obtained by CDMRF; (h) landslide regions obtained by the proposed CDFFCM; and (i-l) landslide mapping results using ELSE, RLSE, CDMRF, and CDFFCM overlaid on the post-event image, respectively. 
Figure 15i-1 shows the comparison results of different approaches for landslide mapping. There were a lot of blue regions in Figure 15i-k, which indicated a great number of true landslide regions were missed by ELSE, RLSE, and CDMRF. The proposed CDFFCM provided better visual effects than three comparative approaches because Figure 151 included more red regions (i.e., true landslide regions) and fewer blue regions (i.e., missing true landslide regions). However, Figure 151 included more pink regions (i.e., detected false landslide regions) as well.

\subsection{Experimental Analysis}

In practical applications, because Precision and Rcall are often dissimilar in some cases, F-score and Accuracy were usually used for evaluating the effect of LM because they are consistent with a subjective evaluation. To illustrate the relationship between the four indices, two examples are presented as follows.

Example 1: Precision and Rcall are similar for performance evaluation of algorithms.

Figure 16 and Table 3 illustrate example 1.

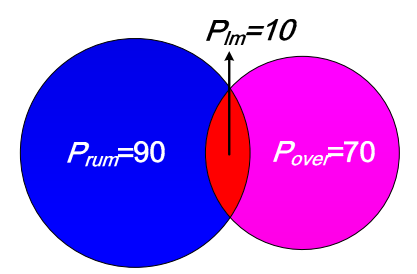

(a)

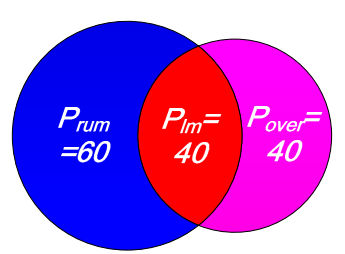

(b)

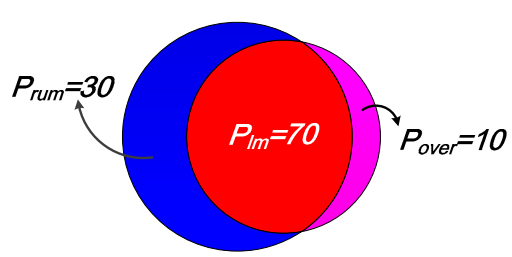

(c)

Figure 16. Example 1 for Precision and Rcall, where $P_{r}=100, P_{l}=80$ : (a) Test 1, (b) Test 2, and (c) Test 3.

Table 3. The variation of Precision and Rcall via increasing $P_{l m}$.

\begin{tabular}{ccccc}
\hline Indices & Test $\mathbf{1}$ & Test 2 & Test 3 & Trend \\
\hline Precision & 0.125 & 0.500 & 0.875 & $\uparrow$ \\
Rcall & 0.100 & 0.400 & 0.700 & $\uparrow$ \\
F-score & 0.111 & 0.444 & 0.778 & $\uparrow$ \\
Accuracy & 0.060 & 0.286 & 0.636 & $\uparrow$ \\
\hline
\end{tabular}

Example 2: Precision and Rcall are dissimilar for performance evaluation of algorithms, i.e., a high Precision corresponds to a low Rcall. However, F-score and Accuracy are consistent larger or smaller.

Generally, $P_{r}$ is a constant while $P_{l}$ is usually a variant. Precision and Rcall are inconsistent when $P_{l}$ decreases as shown in Figure 17 and Table 4.

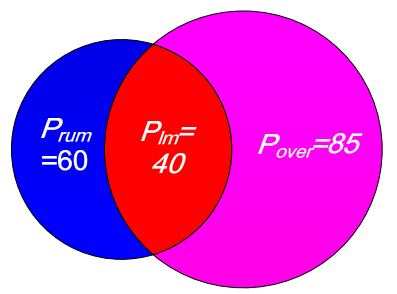

(a)

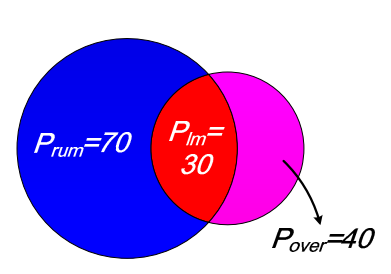

(b)

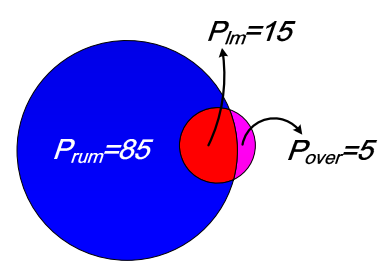

(c)

Figure 17. Example 2 for Precision and Rcall, where $P_{r}=100$ and $P_{l}$ is variant: (a) Test $1, P_{l}=125$, (b) Test 2, $P_{l}=70$, and (c) Test $3, P_{l}=20$. 
Table 4. The variation of Precision and Rcall via decreasing $P_{l}$.

\begin{tabular}{ccccc}
\hline Indices & Test $\mathbf{1}$ & Test $\mathbf{2}$ & Test 3 & Trend \\
\hline Precision & 0.320 & 0.429 & 0.750 & $\uparrow$ \\
Rcall & 0.400 & 0.300 & 0.150 & $\downarrow$ \\
F-score & 0.356 & 0.353 & 0.250 & $\downarrow$ \\
Accuracy & 0.216 & 0.214 & 0.143 & $\downarrow$ \\
\hline
\end{tabular}

Table 4 shows that F-score and Accuracy become larger or smaller consistently but Precision and Rcall were inconsistent. It is thus F-score and Accuracy that were usually used for evaluating the performance of an approach for landslide mapping.

To further verify the effectiveness of the proposed method, the detected landslides were compared with the ground truths by using two popular indices, i.e., F-score and Accuracy. The final quantitative evaluation results are presented in Table 5 and illustrated by a corresponding bar-chart shown in Figure 18 for the four approaches ELSE, RLSE, CDMRF, and the proposed CDFFCM.

Table 5. Quantitative evaluation and comparison of different approaches for landslide mapping.

\begin{tabular}{cccccc}
\hline \multirow{2}{*}{ Study Areas } & Methods & \multicolumn{4}{c}{ Evaluation Indices (\%) } \\
\cline { 3 - 6 } & & Precision & Rcall & F-score & Accuracy \\
\hline \multirow{3}{*}{ A } & ELSE & 90.33 & 51.72 & 65.78 & 49.01 \\
& RLSE & 89.57 & 48.78 & 63.16 & 46.16 \\
& CDMRF & 85.90 & 53.69 & 66.08 & 49.34 \\
& CDFFCM & 79.42 & 93.56 & 85.91 & 75.30 \\
\hline \multirow{3}{*}{ B } & ELSE & 90.69 & 75.93 & 82.66 & 70.44 \\
& RLSE & 90.64 & 72.85 & 80.78 & 67.75 \\
& CDMRF & 88.44 & 77.13 & 82.40 & 70.07 \\
& CDFFCM & 87.86 & 90.26 & 89.04 & 80.25 \\
\hline \multirow{3}{*}{ C } & ELSE & 89.86 & 56.75 & 69.57 & 53.33 \\
& RLSE & 90.17 & 53.59 & 67.22 & 50.63 \\
& CDMRF & 86.25 & 60.10 & 70.84 & 54.84 \\
& CDFFCM & 76.18 & 81.66 & 78.82 & 65.05 \\
\hline
\end{tabular}

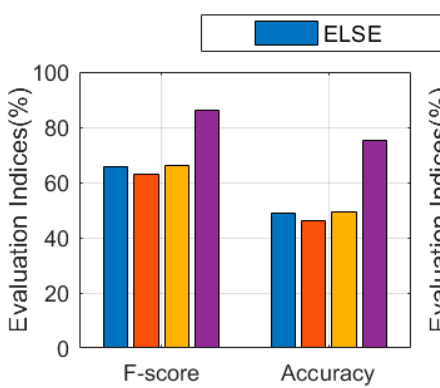

(a)

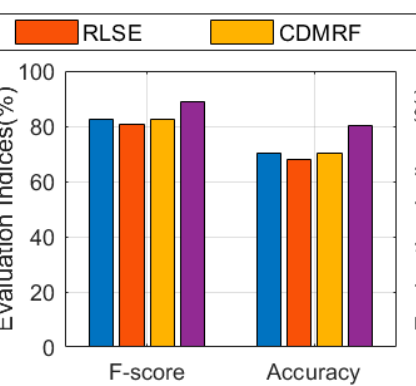

(b)

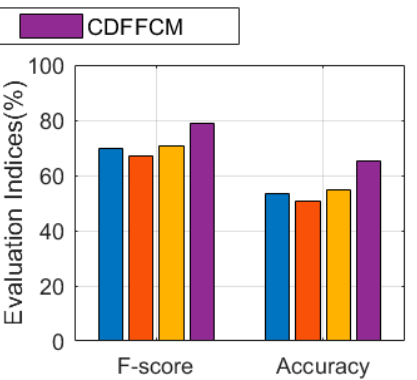

(c)

Figure 18. Bar-chart corresponding to the values of Table 5: (a) A-area, (b) B-area, and (c) C-area.

For A-area, B-area, and C-area, ELSE, RLSE, and CDMRF provide higher Precision but lower Recall than CDFFCM because a larger number of true landslide regions were missed by the first three approaches, i.e., the first three approaches obtained a small value of $P_{l}$ while CDFFCM obtained a large value of $P_{l}$. However, the proposed CDFFCM obtained a significantly higher F-score and Accuracy than the three other approaches shown in Table 5, which demonstrates that CDFFCM was superior to the three comparative approaches on three study areas. 
The four approaches obtain higher Precision and Recall for B-area than A-area and C-area because the landslide regions were covered by more grass and fewer trees in B-area than in A-area and C-area. Trees led to the missing of more true landslide regions than grass. Consequently, there were less pink regions in Figure 141 than Figures 131 and 15l, and B-area obtained higher values with F-score and Accuracy than A-area and C-area.

To further demonstrate that the proposed CDFFCM had a high computational efficiency for LM, Table 6 shows the comparison of the execution time of these different approaches on A-area, B-area, and C-area. The four approaches were implemented repeatedly more than thirty times, the final average execution time is shown in Table 6 . The proposed approach required a shorter execution time than the three comparative approaches. The running time of ELSE, RLSE, and CDMRF relied on the image size. For example, ELSE required $80.12 \mathrm{~s}$ for A-area but $29.32 \mathrm{~s}$ for B-area since the size of A-area was $1252 \times 2199$ but $923 \times 593$ for B-area. The computational complexity of the proposed CDFFCM did not depend on the image size because the Gaussian pyramid method is able to obtain difference resolution images. The fuzzy clustering algorithm was only performed on a low-resolution image to obtain clustering centers. Therefore, the proposed CDFFCM had the advantage of a faster execution time.

In Table 6, A-area required a longer execution time than B-area and C-area for all four approaches because the size of A-area was larger than B-area and C-area. The proposed CDFFCM required a shorter execution time than the three comparative approaches because it employed the fast FCM algorithm to achieve image segmentation. Therefore, CDFFCM was clearly superior to the three comparative approaches due to the high computational efficiency, as shown in Figure 19.

Table 6. Comparison of execution time of four different approaches (in seconds).

\begin{tabular}{ccccc}
\hline \multirow{2}{*}{ Study Areas } & \multicolumn{4}{c}{ Methods } \\
\cline { 2 - 5 } & ELSE & RLSE & CDMRF & CDFFCM \\
\hline A & 80.12 & 57.21 & 80.10 & 16.92 \\
B & 29.32 & 39.41 & 29.31 & 11.20 \\
C & 51.98 & 48.03 & 43.52 & 13.57 \\
\hline
\end{tabular}

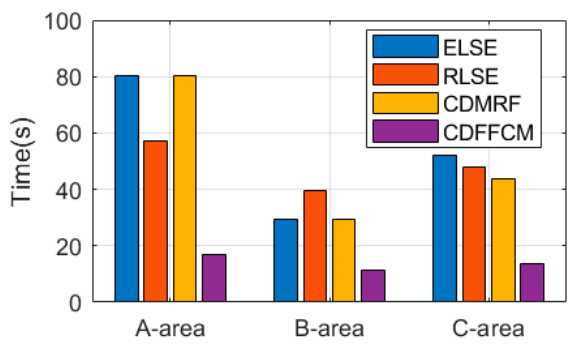

Figure 19. Bar-chart corresponding to the values in Table 6.

\section{Discussion}

In the paper, the proposed CDFFCM was evaluated for different landslide images under appropriate parameter settings. The CDFFCM included two required parameters, where $T_{1}$ was a threshold used for comparing with the values of clustering centers, and it was used for image binarization as well. $c$ was the number of clusters used for FCM. The structuring element denoted by $B$ was used for both image filtering and morphological reconstruction, where $r$ was the radius of structuring element and it could be decided by the image size. The multiple of downsampling denoted by $p$, was used for the Gaussian pyramid. For the proposed CDFFCM, $T_{1}$ and $c$ were required, but $r$ and $p$ were unrequired because the latter two parameters could be computed automatically according to the size of the bitemporal images, i.e., 


$$
p=\operatorname{round}(\min (W, H) / 200) \text {. }
$$

The parameter $r$ could be computed according to Equation (12). Therefore, the proposed CDFFCM only required setting the values of $T_{1}$ and $c$.

To test the influence of parameters on the performance of CDFFCM, four quantitative evaluation indices, Precision, Recall, F-score, and Accuracy were analyzed. Taking B-area as the research object and setting $c=5$, Figure 20a shows the effect of a change on each index by setting different values of $T_{1}$. A small value of $T_{1}$ meant too many false landslide regions were detected from the pre-event image, which indicated the detected-true landslide regions were much smaller than the ground truth. Consequently, a high Precision and low Recall, F-score, and Accuracy were obtained in Figure 18a. By increasing $T_{1}$, the detected result of false landslide regions was closer to the true result, which indicated the detected-true landslide regions were closer to the ground truth. Therefore, the values of Recall, F-score, and Accuracy got significantly larger, while the value of Precision got slightly smaller, as shown in Figure 20a. Furthermore, we can see that the four indices were convergent when $T_{1} \geq 0.6$. A similar experiment was carried out on A-area and C-area. We obtained $T_{1} \geq 0.65$. Therefore, we considered $T_{1}=0.8$ as an empirical value in our experiments.

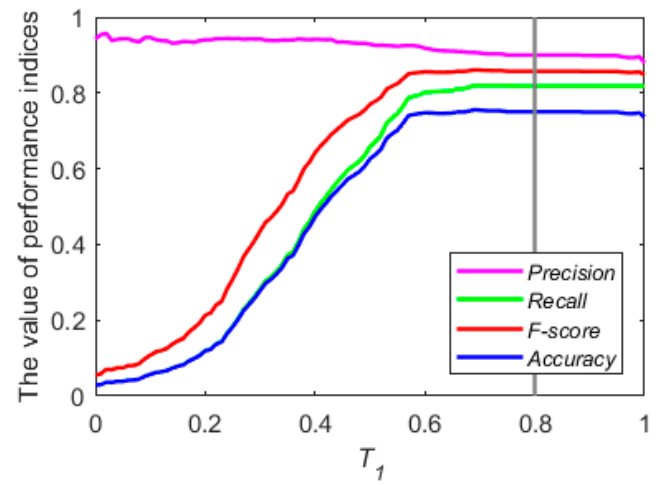

(a)

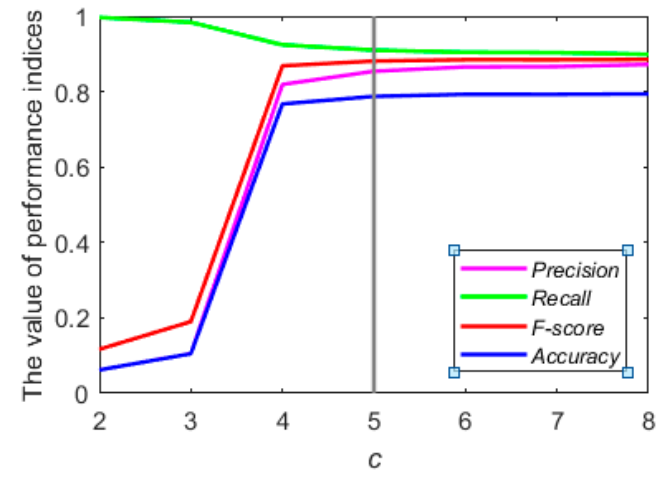

(b)

Figure 20. The influence of parameters on the performance of the proposed CDFFCM: (a) parameter $T_{1}$, and (b) parameter $c$.

Figure 20a shows the influence of on the performance of CDFFCM. Similarly, we analyzed the influence of $c$ on the performance of CDFFCM, as shown in Figure 20b. When the value of $c$ was small, the detected landslide regions were much larger than the ground truth, resulting in a high Recall and low Precision, F-score, and Accuracy. With the increase of the value of $c$, the four indices were convergent, and higher values of F-score and Accuracy were obtained. Because a large $c$ would lead to a high computational complexity for FCM algorithm, we considered $c=5$ as an empirical value in our experiments.

In our experiments, we detected the landslides using bitemporal images, which were acquired at different times. Because the difference image of the bitemporal images mainly included landslides but no other changed information such as land use change, forest deformation, and lake water level change event, etc., the proposed CDFFCM provided a high accuracy of landslide identification in our experiments. However, if the post-event image included other changed information, for example, a new road, it will be difficult to differentiate a new road and landslides because they have similar image features. Therefore, the performance of the proposed CDFFCM relies on the bitemporal images. If we want to identify landslides from changed regions, a classifier is required, and a great number of training samples and label images are necessary as well. However, the collection of a great number of bitemporal images and corresponding label images is difficult to us to obtain, therefore we did not give much discussion about the classification of changed regions. 


\section{Conclusions}

A change detection approach using fast fuzzy c-means clustering for landslide mapping (CDFFCM) has been presented in this paper. The proposed CDFFCM addresses two problems that exist in popular approaches for LM, such as ELSE, RLSE, and CDMRF. On the one hand, the existing approaches mainly depend on the illumination change of bitemporal images but miss the spatial structuring information of images, which leads to a serious landslide region leak. The proposed CDFFCM employs an improved FCM algorithm that incorporates the local spatial information into its objective function to obtain a better difference image. On the other hand, the existing approaches require a long execution time for VHR images. The proposed CDFFCM utilizes a Gaussian pyramid method to reduce the redundant data of images for FCM to improve its computational efficiency. The proposed CDFFCM has been applied to a landslide site with A-area, B-area, and C-area, on Lantau Island, Hong Kong, China. The images of the three areas have a different size, where A-area was larger than B-area. The experimental results demonstrate that the proposed CDFFCM was effective and efficient for LM since the two problems mentioned above were successfully addressed.

Though the proposed CDFFCM was efficient and effective for landslide mapping from VHR remote sensing images, two parameters must be set manually. For future studies, additional remote sensing images with different types of landslides will be collected and applied to verify the performance of the proposed CDFFCM. In addition, it is still a challenge to identify landslides from changed regions when the post-event image includes a complex change information for the proposed CDFFCM. With the development of convolutional neural network (CNN), we try to employ CNN to achieve end-to-end landslide mapping. Therefore, we need to collect more landslide images and corresponding ground truths.

Author Contributions: T.L. and Y.Z. provided the original idea for the study, Z.L. and S.L. conceived and designed the experiments, D.X. and S.L. performed the experiments, Z.L. and D.X. analyzed the data, T.L. wrote the paper, and A.K.N. revised the paper. All authors read and approved the final manuscript. Z.L. has the equal contribution as the first author.

Funding: This work was supported in part by the National Natural Science Foundation of China under Grant 61461025, Grant 61871259, Grant 61811530325, Grant 61701396, Grant 61701387, in part by China Postdoctoral Science Foundation under Grant 2016M602856, and in part by the National Science Foundation of Shanghai under grant 16JC1401300.

Acknowledgments: The authors thank the editor-in-thief, associate editor, and reviewers for their insightful comments and valuable suggestions.

Conflicts of Interest: The authors declare no conflict of interest.

\section{References}

1. Chander, G.; Hewison, T.J.; Fox, N.; Wu, X.; Xiong, X.; Blackwell, W.J. Overview of intercalibration of satellite instruments. IEEE Trans. Geosci. Remote Sens. 2013, 51, 1056-1080. [CrossRef]

2. Komarov, A.; Buehner, M. Automated detection of ice and open water from dual-polarization RADARSAT-2 images for data assimilation. IEEE Trans. Geosci. Remote Sens. 2017, 55, 5755-5769. [CrossRef]

3. Pacifici, F.; Longbotham, N.; Emery, W.J. The importance of physical quantities for the analysis of multitemporal and multiangular optical very high spatial resolution images. IEEE Trans. Geosci. Remote Sens. 2014, 52, 6241-6256. [CrossRef]

4. Cartus, O.; Siqueira, P.; Kellndorfer, J. An error model for mapping forest cover and forest cover change using L-Band SAR. IEEE Geosci. Remote Sens. Lett. 2017, 15, 107-111. [CrossRef]

5. Zhan, X.; Zhang, R.; Wang, P.; Huang, C.; Csiszar, I.; Zhou, L.; Weng, F. Monitoring surface type changes with S-NPP/JPSS VIIRS observations. In Proceedings of the 2017 IEEE International Geoscience and Remote Sensing Symposium (IGARSS), Fort Worth, TX, USA, 23-28 July 2017; pp. 1288-1291.

6. Nelson, R.F. Detecting forest canopy change due to insect activity using landsat MSS. Photogramm. Eng. Remote Sens. 1983, 49, 1303-1314. 
7. Jin, Y. Change detection of enhanced, no-changed and reduced scattering in multi-temporal ERS-2 SAR images using the two-thresholds EM and MRF algorithms. In Proceedings of the 2005 IEEE International Geoscience and Remote Sensing Symposium (IGARSS 2005), Seoul, Korea, 29-29 July 2005; pp. 3994-3997.

8. Lv, Z.; Shi, W.; Zhang, X.; Benediktsson, J.A. Landslide inventory mapping from bitemporal high-resolution remote sensing images using change detection and multiscale segmentation. IEEE J. Sel. Top. Appl. Earth Obs. Remote Sens. 2018, 11, 1520-1532. [CrossRef]

9. Li, Z.; Shi, W.; Lu, P.; Hao, M.; Yan, L.; Wang, Q.; Miao, Z. Landslide mapping from aerial photographs using change detection-based Markov random field. Remote Sens. Environ. 2016, 187, 76-90. [CrossRef]

10. Li, Z.; Shi, W.; Myint, S.W.; Lu, P.; Wang, Q. Semi-automated landslide inventory mapping from bitemporal aerial photographs using change detection and level set method. Remote Sens. Environ. 2016, 175, 215-230. [CrossRef]

11. Dong, R.; Wang, H. A novel VHR image change detection algorithm based on image fusion and fuzzy c-means clustering. arXiv, 2017; arXiv:1706.07157.

12. Li, Z.; Shi, W.; Zhang, H.; Hao, M. Change detection based on gabor wavelet features for very high resolution remote sensing images. IEEE Geosci. Remote Sens. Lett. 2017, 14, 783-787. [CrossRef]

13. Zheng, Y.; Jiao, L.; Liu, H.; Zhang, X.; Hou, B.; Wang, S. Unsupervised saliency-guided SAR image change detection. Pattern Recognit. 2017, 61, 309-326. [CrossRef]

14. Shi, W.; Shao, P.; Hao, M.; Hao, M.; He, P.; Wang, J. Fuzzy topology-based method for unsupervised change detection. Remote Sens. Lett. 2016, 7, 81-90. [CrossRef]

15. Shao, P.; Shi, W.; He, P.; Hao, M.; Zhang, X. Novel approach to unsupervised change detection based on a robust semi-supervised FCM clustering algorithm. Remote Sens. 2016, 8, 264. [CrossRef]

16. Sharma, A.; Gulati, T. Change detection in remotely sensed images based on image fusion and fuzzy clustering. Int. J. Electron. Eng. Res. 2017, 9, 141-150.

17. Gong, M.; Zhou, Z.; Ma, J. Change detection in synthetic aperture radar images based on image fusion and fuzzy clustering. IEEE Trans. Image Process. 2012, 21, 2141-2151. [CrossRef] [PubMed]

18. Ghosh, A.; Mishra, N.S.; Ghosh, S. Fuzzy clustering algorithms for unsupervised change detection in remote sensing images. Inform. Sci. 2011, 181, 699-715. [CrossRef]

19. Gong, M.; Su, L.; Jia, M.; Chen, W. Fuzzy clustering with a modified MRF energy function for change detection in synthetic aperture radar images. IEEE Trans. Fuzzy Syst. 2014, 22, 98-109. [CrossRef]

20. Zhang, X.; Xiao, P.; Feng, X.; Yuan, M. Separate segmentation of multi-temporal high-resolution remote sensing images for object-based change detection in urban area. Remote Sens. Environ. 2017, 201, $243-255$. [CrossRef]

21. Keyport, R.N.; Oommen, T.; Martha, T.R.; Sajinkumar, K.S.; Gierke, J.S. A comparative analysis of pixel-and object-based detection of landslides from very high-resolution images. Int. J. Appl. Earth Obs. Geoinf. 2018, 64, 1-11. [CrossRef]

22. Lv, Z.; Liu, T.; Wan, Y.; Benediktsson, J.A.; Zhang, X. Post-processing approach for refining raw land cover change detection of very high-resolution remote sensing images. Remote Sens. 2018, 10, 472. [CrossRef]

23. Leichtle, T.; Geiß, C.; Wurm, M.; Lakes, T.; Taubenböck, H. Unsupervised change detection in VHR remote sensing imagery-An object-based clustering approach in a dynamic urban environment. Int. J. Appl. Earth Obs. Geoinf. 2017, 54, 15-27. [CrossRef]

24. Wang, Q.; Yuan, Y.; Du, Q. GETNET: A general end-to-end two-dimensional CNN framework for hyperspectral image change detection. IEEE Trans. Geosci. Remote Sens. 2018, 99. [CrossRef]

25. Yu, H.; Yang, W.; Hua, G.; Ru, H.; Huang, P. Change detection using high resolution remote sensing images based on active learning and markov random fields. Remote Sens. 2017, 9, 1233. [CrossRef]

26. Lv, Z.; Zhang, W. Contextual analysis based approach for detecting change from high resolution satellite imagery. J. Indian Soc. Remote Sens. 2018, 46, 43-50. [CrossRef]

27. Park, N.W.; Chi, K.H. Quantitative assessment of landslide susceptibility using high-resolution remote sensing data and a generalized additive model. Int. J. Remote Sens. 2008, 29, 247-264. [CrossRef]

28. Lu, P.; Stumpf, A.; Kerle, N.; Casagli, N. Object-oriented change detection for landslide rapid mapping. IEEE Geosci. Remote Sens. Lett. 2011, 8, 701-705. [CrossRef]

29. Van, A.W.; Batenburg, K.J.; Sijbers, J. Optimal threshold selection for segmentation of dense homogeneous objects in tomographic reconstructions. IEEE Trans. Med. Imaging 2011, 30, 980-989. 
30. Huo, G.; Yang, S.X.; Li, Q.; Zhou, Y. A robust and fast method for sidescan sonar image segmentation using nonlocal despeckling and active contour model. IEEE Trans. Cybern. 2017, 47, 855-872. [CrossRef] [PubMed]

31. Bai, M.; Urtasun, R. Deep watershed transform for instance segmentation. In Proceedings of the IEEE Conference on Computer Vision and Pattern Recognition, Honolulu, HI, USA, 22-25 July 2017; pp. 2858-2866.

32. Grady, L. Random walks for image segmentation. IEEE Trans. Pattern Anal. Mach. Intell. 2006, 8, 1768-1783. [CrossRef] [PubMed]

33. Ju, W.; Xiang, D.; Zhang, B.; Chen, C. Random walk and graph cut for co-segmentation of lung tumor on PET-CT images. IEEE Trans. Image Process. 2015, 24, 5854-5867. [CrossRef] [PubMed]

34. Chen, J.; Li, Z.; Huang, B. Linear spectral clustering superpixel. IEEE Trans. Image Process. 2017, 26, 3317-3330. [CrossRef] [PubMed]

35. Ji, Z.; Liu, J.; Cao, G.; Sun, Q.; Chen, Q. Robust spatially constrained fuzzy c-means algorithm for brain MR image segmentation. Pattern Recognit. 2014, 47, 2454-2466. [CrossRef]

36. Ji, Z.; Huang, Y.; Xia, Y.; Zheng, Y. A robust modified Gaussian mixture model with rough set for image segmentation. Neurocomputing 2017, 266, 550-565. [CrossRef]

37. Shelhamer, E.; Long, J.; Darrell, T. Fully convolutional networks for semantic segmentation. IEEE Trans. Pattern Anal. Mach. Intell. 2017, 39, 640-651. [CrossRef] [PubMed]

38. Gu, J.; Jiao, L.; Yang, S.; Liu, F. Fuzzy double c-means clustering based on sparse self-representation. IEEE Trans. Fuzzy Syst. 2018, 26, 612-626. [CrossRef]

39. Yang, J.; He, Y.; Caspersen, J. Region merging using local spectral angle thresholds: A more accurate method for hybrid segmentation of remote sensing images. Remote Sens. Environ. 2017, 190, 137-148. [CrossRef]

40. Wang, Q.; He, X.; Li, X. Locality and structure regularized low rank representation for hyperspectral image classification. IEEE Trans. Geosci. Remote Sens. 2018. [CrossRef]

41. Nie, F.; Wang, X.; Jordan, M.I.; Huang, H. The constrained laplacian rank algorithm for graph-based clustering. In Proceedings of the Association for the Advance of Artificial Intelligence, Phoenix, AZ, USA, 12-17 February 2016; pp. 1969-1976.

42. Ding, S.; Jia, H.; Du, M.; Xue, Y. A semi-supervised approximate spectral clustering algorithm based on HMRF model. Inform. Sci. 2018, 429, 215-228. [CrossRef]

43. Yang, M.; Nataliani, Y. Robust-learning fuzzy c-means clustering algorithm with unknown number of clusters. Pattern Recognit. 2017, 71, 45-59. [CrossRef]

44. Szilagyi, L.; Benyo, Z.; Szilagyii, S.M.; Adam, H.S. MR brain image segmentation using an enhanced fuzzy c-means algorithm. In Proceedings of the IEEE Engineering in Medicine and Biology Society, Cancun, Mexico, 17-21 September 2003; pp. 17-21.

45. Lei, T.; Jia, X.; Zhang, Y.; He, L.; Meng, H.; Nandi, A.K. Significantly fast and robust fuzzy c-means clustering algorithm based on morphological reconstruction and membership filtering. IEEE Trans. Fuzzy Syst. 2018. [CrossRef]

46. Lan, Z.; Lin, M.; Li, X.; Hauptmann, A.G.; Raj, B. Beyond gaussian pyramid: Multi-skip feature stacking for action recognition. In Proceedings of the IEEE Conference on Computer Vision and Pattern Recognition, Boston, MA, USA, 8-10 June 2015; pp. 204-212.

47. Zhang, H.; Wang, Q.; Shi, W.; Hao, M. A novel adaptive fuzzy local information c-means clustering algorithm for remotely sensed imagery classification. IEEE Trans. Geosci. Remote Sens. 2017, 55, 5057-5068. [CrossRef]

48. Memon, K.H.; Lee, D.H. Generalised fuzzy c-means clustering algorithm with local information. IET Image Process. 2017, 11, 1-12. [CrossRef]

49. Guo, F.; Wang, X.; Shen, J. Adaptive fuzzy c-means algorithm based on local noise detecting for image segmentation. IET Image Process. 2016, 10, 272-279. [CrossRef]

50. Lei, T.; Zhang, Y.; Wang, Y.; Liu, S.; Guo, Z. A conditionally invariant mathematical morphological framework for color images. Inform. Sci. 2017, 387, 34-52. [CrossRef]

51. Chen, J.J.; Su, C.; Grimson, W.E.L.; Liu, J.; Shiue, D.H. Object segmentation of database images by dual multiscale morphological reconstructions and retrieval applications. IEEE Trans. Image Process. 2012, 21, 828-843. [CrossRef] [PubMed]

(C) 2018 by the authors. Licensee MDPI, Basel, Switzerland. This article is an open access article distributed under the terms and conditions of the Creative Commons Attribution (CC BY) license (http://creativecommons.org/licenses/by/4.0/). 